\title{
EMISSÕES OTOACÚSTICAS PRODUTO DE DISTORÇÃO EM NORMO OUVINTES E EM PERDAS AUDITIVAS NEUROSSENSORIAIS LEVE E MODERADA COM OS PROTOCOLOS 65/55 DB NPS E 70/70 DB NPS
}

\author{
Distortion product otoacoustic emission in audilogically \\ normal people and in mild and moderate sensorineural hearing loss \\ with 65/55 dBSPL and 70/70 dBSPL protocols
}

\author{
Maria Isabel Kós ${ }^{(1)}$, Kátia de Almeida ${ }^{(2)}$, Silvana Frota ${ }^{(3)}$, Ana Cristina Hiromi Hoshino ${ }^{(4)}$
}

\section{RESUMO}

Objetivo: estudar comparativamente as amplitudes das emissões otoacústicas produto de distorção em indivíduos normo ouvintes, com perdas auditivas de graus leve e moderado. Métodos: a amostra compôs-se de 41 indivíduos na faixa etária de 18 a 45 anos, sendo 25 do sexo feminino e 16 do sexo masculino. Foram realizadas audiometrias tonal e vocal para classificar os grupos conforme perfil audiométrico; imitanciometria para avaliar as condições da orelha média; e emissões otoacústicas produto de distorção, utilizando os protocolos $\mathrm{L} 1=65 \mathrm{~dB}$ NPS e $\mathrm{L} 2=55 \mathrm{~dB}$ NPS e $\mathrm{L} 1=\mathrm{L} 2=70 \mathrm{~dB}$ NPS. No total, foram analisadas 63 orelhas (32 orelhas direitas e 31 orelhas esquerdas). Os indivíduos selecionados foram distribuídos em três grupos distintos, sendo o primeiro grupo (G1) composto de 21 orelhas com audiometria tonal com limiares dentro da normalidade (grupo controle); o segundo grupo (G2) composto de 21 orelhas com perdas auditivas neurossensoriais de grau leve; e o terceiro grupo (G3) composto por 21 orelhas com perdas auditivas neurossensoriais de grau moderado. Os dados audiométricos foram comparados com os resultados das Emissões Otoacústicas Produto de Distorção (DPOAE). Resultados: encontrou-se diminuição e ausência das amplitudes no grupo com perda auditiva de grau leve (G2) e ausência de respostas no grupo com perda auditiva de grau moderado (G3) em ambos os protocolos. Conclusão: com relação ao grau da perda auditiva, pode-se concluir que quanto maior a perda auditiva, menor o aumento da amplitude das EOAPD.

DESCRITORES: Perda Auditiva; Amplitude; Emissões Otoacústicas Espontâneas

(1) Fonoaudióloga; Professora Auxiliar do Curso de Fonoaudiologia da Universidade Federal do Rio de Janeiro, UFRJ, Rio de Janeiro, RJ; Mestre em Fonoaudiologia pela Universidade Veiga de Almeida.

(2) Fonoaudióloga; Professora Adjunta do Curso de Fonoaudiologia da Santa Casa de Misericórdia de São Paulo, SCMSP, São Paulo, SP; Professora do Curso de Mestrado em Fonoaudiologia da Universidade Veiga de Almeida, UVA, Rio de Janeiro, RJ; Doutora em Distúrbios da Comunicação Humana pela Universidade Federal de São Paulo.

(3) Fonoaudióloga; Professora Adjunta do Curso de Fonoaudiologia da Universidade Federal do Rio de Janeiro, UFRJ, Rio de Janeiro, RJ; Professora do Curso de Mestrado em Fonoaudiologia da Universidade Veiga de Almeida, UVA, Rio de Janeiro, RJ; Doutora em Distúrbios da Comunicação Humana pela Universidade Federal de São Paulo.

(4) Fonoaudióloga da Clínica Otorrino Barra, Rio de Janeiro, RJ; Mestre em Saúde Coletiva pelo Instituto de Estudos em Saúde Coletiva da Universidade Federal do Rio de Janeiro. Conflito de interesse: inexistente

\section{INTRODUÇÃO}

Uma avaliação e um diagnóstico preciso e precoce são a essência de uma intervenção primária no sentido da prevenção da doença, fazendo com que a população tenha não apenas saúde, mas qualidade de vida. Para o diagnóstico da deficiência auditiva, é recomendado que se realize uma bateria de testes, utilizando tanto avaliações comportamentais como eletrofisiológicas ${ }^{1}$.

O registro das Emissões Otoacústicas Evocadas (EOAE) é o método mais recente para detecção das alterações auditivas de origem coclear. Consiste em um método objetivo, relativamente simples, rápido e não invasivo, o qual dispensa o uso de eletrodos 
e que pode ser realizado em qualquer faixa etária, ressaltando-se sua aplicação na rotina clínica ${ }^{2}$. Encontra-se Emissões Otoacústicas (EOA) presentes em todas as orelhas funcionalmente normais $\mathrm{e}$ que deixam de ser detectadas, quando os limiares tonais forem superiores a 20-30 dB ${ }^{2}$.

As emissões otoacústicas transientes (EOAT) são ainda as mais utilizadas tanto para realizar a triagem auditiva neonatal, como na avaliação clínica. Acredita-se que esta possui parâmetros de registros mais definidos do que a emissões otoacústicas produto de distorção (EOAPD) ${ }^{3}$. Por outro lado, a EOAPD possui características importantes para um diagnóstico mais preciso e precoce da deficiência auditiva, tais como: a análise por bandas de frequência, variação do tipo e da intensidade de estímulo sonoro. Pode-se variar a intensidade dos níveis de estímulos utilizando protocolos como $\mathrm{L} 1=\mathrm{L} 2=70 \mathrm{~dB}$ NPS ou $\mathrm{L} 1=65 \mathrm{~dB}$ NPS/L2=55 dB NPS. Os estímulos de menor intensidade serão mais sensíveis e precisos no diagnóstico das perdas auditivas neurossensoriais de graus entre leve e moderado. A partir desses parâmetros, cria-se a possibilidade do teste de EOAPD auxiliar no diagnóstico diferencial entre as perdas auditivas neurossensoriais leve e moderada ${ }^{2}$.

$\mathrm{Na}$ pesquisa das EOAPD, quando realizada em lactentes como procedimento de triagem auditiva, 0 protocolo mais utilizado é $L 1=65 \mathrm{~dB}$ NPS/L2 $=55 \mathrm{~dB}$ NPS, pois se torna o exame mais sensível para diagnosticar perdas auditivas sensorioneurais leves ${ }^{3}$. Outra vantagem de se utilizar este protocolo é que a correlação com os limiares audiométricos é mais fidedigna, quando comparado com o protocolo $\mathrm{L} 1=\mathrm{L} 2=70 \mathrm{~dB}$ NPS, tanto para exames normais como para perdas auditivas ${ }^{4}$.

As formas de avaliação das respostas das EOAPD também possuem uma variedade maior de análise. Um parâmetro simples, rápido que não requer outro procedimento é a comparação da amplitude das respostas das EOAPD. Sabe-se que o próprio exame de EOA já está consagrado pela sua vantagem de detectar alterações nas células ciliadas externas (CCE), antes mesmo da audiometria tonal e vocal. Pode-se também afirmar que a amplitude das respostas pode variar nos casos em que as EOAT e as EOAPD estão presentes ${ }^{4}$.

Existem estudos relacionando o grau da perda auditiva com a amplitude das Emissões Otoacústicas ${ }^{5,6}$. Em outros estudos, observa-se que a imaturidade do recém-nascido ou a prematuridade deste pode interferir na EOA. Em ambos os casos, foi observada uma diminuição das amplitudes seguida de normalidade, após o reteste, caracterizando a importância deste parâmetro na triagem auditiva neonatal ${ }^{7,8}$.
Indivíduos expostos a sons intensos ou a agentes ototóxicos podem apresentar mudanças na amplitude das EOA. Estudos relatam que, quando existem alterações de CCE, mesmo que ainda não exista mudança dos limiares tonais, já é possível observar uma redução da amplitude das EOA, demonstrando a sensibilidade deste parâmetro no diagnóstico precoce e/ou acompanhamento nos tratamentos quimioterápicos bem como nas questões de saúde do trabalhador ${ }^{9-12}$.

Este estudo teve como objetivo estudar comparativamente as amplitudes das EOAPD em indivíduos normo ouvintes, com perdas auditivas de graus leve e moderado, utilizando os protocolos $\mathrm{T} 1$ (L1 = 65 dB NPS e L2 = 55 dB NPS) e T2 (L1=L2=70 dB NPS).

\section{MÉTODOS}

O presente estudo de caráter epidemiológico descritivo foi desenvolvido na Universidade Veiga de Almeida, por meio do estudo da função coclear de 41 indivíduos encaminhados por diversos serviços médicos da região. A faixa etária estipulada foi de 18 a 45 anos e não houve determinação com relação à variável sexo. Utilizou-se como critério de inclusão a presença de audição dentro da normalidade ou perda auditiva neurossensorial leve/ moderada e critérios de exclusão, alterações de orelha externa e/ou média, alterações nos exames imitanciométrico e de potencial evocado auditivo de tronco encefálico (PEATE).

Foram selecionados para participar da pesquisa 41 indivíduos adultos, sendo 25 do sexo feminino e 16 do sexo masculino; 12 indivíduos apresentaram audiometria tonal e vocal com limiares dentro da normalidade e 29 indivíduos apresentaram perdas auditivas neurossensoriais de grau leve ou moderado, unilateral ou bilateral, com idades variando de 18 a 45 anos. No total, foram analisadas 63 oreIhas (32 orelhas direitas e 31 orelhas esquerdas). Os indivíduos selecionados foram divididos em três grupos distintos, sendo o primeiro grupo (G1) composto de 21 orelhas com audiometria tonal com limiares dentro da normalidade (grupo controle); o segundo grupo (G2) composto de 21 orelhas com perdas auditivas neurossensoriais de grau leve e 0 terceiro grupo (G3) composto por 21 orelhas com perdas auditivas neurossensoriais de grau moderado. Todos os indivíduos foram submetidos à:

1) Avaliação otorrinolaringológica, com o objetivo de excluir a presença de alterações de orelha externa e/ou média e retirada de cerume, sempre que necessário; 
2) Imitanciometria, com a finalidade de descartar possíveis problemas de orelha média, que pudessem interferir no resultado do exame;

3) Audiometria tonal liminar e vocal para classificar os grupos, segundo padrão de classificação ${ }^{13}$ : 0 - $20 \mathrm{~dB}$ NA - normal; 21 - $40 \mathrm{~dB}$ NA perda auditiva leve; 41 - $70 \mathrm{~dB}$ NA - perda auditiva moderada;

4) Potencial Evocado Auditivo de Tronco Encefálico para excluir uma possível alteração retrococlear por meio do estudo das ondas I, III e V. Para realizar o exame, foi utilizado um equipamento de Respostas Elétricas de Tronco Encefálico, modelo 904, versão 7,26 da marca Bio-logic.

Os sujeitos selecionados foram submetidos à pesquisa das Emissões Otoacústicas Produto de Distorção. Foi usado um equipamento de pesquisa das emissões otoacústicas produto de distorção Scout Plus, Version 7,26, Navigator Pró da marca Bio-logic, acoplado a um computador Pentium II. $O$ registro das EOAPD foi realizado com os indivíduos deitados em uma maca, em uma sala tratada acusticamente. Foi utilizado para captar os sinais um microfone miniaturizado alojado no meato acústico externo, vedado por uma oliva de látex ou de espuma.

Os exames de EOAPD foram realizados em dois momentos distintos denominados T1 e T2. Em T1 o protocolo utilizado foi $\mathrm{L} 1=\mathrm{L} 2 \mathrm{em}$ que $\mathrm{L} 1=65 \mathrm{~dB}$ NPS e L2 $=55 \mathrm{~dB}$ NPS dB, já no T2, o protocolo foi $\mathrm{L} 1=\mathrm{L} 2=70 \mathrm{~dB}$ NPS.

Foram analisadas as médias geométricas (GM) nas frequências de $1.797 \mathrm{~Hz}, 2.566 \mathrm{~Hz}, 3.640 \mathrm{~Hz}$, $5.133 \mathrm{~Hz}$ e $7.280 \mathrm{~Hz}$.

As EOAPD foram consideradas como presentes quando registradas pelo menos $3 \mathrm{~dB}$ e $6 \mathrm{~dB}$ acima do ruído de fundo (NF) e considerado exame normal quando pelo menos quatro das cinco frequências estiveram presentes.

Para comparar as médias geométricas (GM) encontradas nos exames de EOAPD, nas frequências de $1.797 \mathrm{~Hz}, 2.566 \mathrm{~Hz}, 3.640 \mathrm{~Hz}$, $5.133 \mathrm{~Hz}$ e $7.280 \mathrm{~Hz}$, e na audiometria tonal liminar por via aérea, utilizou-se as frequências de: $2.000 \mathrm{~Hz}, 3.000 \mathrm{~Hz}, 4.000 \mathrm{~Hz}, 6.000 \mathrm{~Hz}$ e $8.000 \mathrm{~Hz}$ (Tabela 1).

O estudo considerou os aspectos éticos recomendados pela Resolução 196/96 sobre Pesquisa envolvendo seres humanos, incluindo entre outros, a obtenção do Consentimento Livre e Esclarecido dos indivíduos. Assegurou que a participação não acarretaria nada que pudesse levar a danos físicos, psíquicos, morais, intelectuais, sociais, culturais ou espirituais dessas pessoas. O estudo foi avaliado e aprovado (Processo n. 32/2004) pelo Comitê de Ética da instituição.

Ao final da pesquisa todos os indivíduos receberam um relatório contendo todos os resultados dos testes audiológicos a que foram submetidos.

Foram calculadas as medidas de tendência de medida central (TC) para os resultados da Audiometria Tonal liminar e para as amplitudes das Emissões Otoacústicas Produto de Distorção em T1 $(\mathrm{L} 1=65 \mathrm{~dB}$ NPS e L2 $=55 \mathrm{~dB}$ NPS) e T2 (L1 = $\mathrm{L} 2=70 \mathrm{~dB}$ NPS).

Para comparação das amplitudes das EOAPD, avaliando os dados de forma quantitativa, entre os diferentes grupos, comparando G1 (grupo controle), G2 (grupo de indivíduos com perdas auditivas neurossensoriais de grau leve) e G3 (grupo de indivíduos com perdas auditivas neurossensoriais de grau moderado), foi realizada a Análise de Variância de Kruskal-Wallis.

Para analisar a variação das amplitudes dos G1, G2 e G3 entre os testes T1 e T2 foi aplicado o teste dos postos sinalizados de Wilcoxon. Foram utilizados métodos não paramétricos, pois as medidas de amplitudes das EOAPD não apresentaram distribuição normal (distribuição Gaussiana) devido à dispersão dos dados e à falta de simetria da distribuição.

O critério de determinação de significância adotado foi o nível de $5 \%$, ou seja, quando o valor de $p$ do teste estatístico for menor ou igual a 0,05, então existe significância estatística, assinalando-se os valores significantes com asterisco. A análise estatística foi processada pelo software estatístico $S A S^{\circledR}$ System.

\section{RESULTADOS}

\section{Análise das amplitudes EOAPD nos grupos G1, G2 e G3}

A Tabela 1 fornece as médias, desvios padrões (DP), segundo os grupos de indivíduos (G1, G2 e G3) e o correspondente nível descritivo do teste estatístico para as intensidades dos protocolos T1 e T2, respectivamente. Esta análise teve como objetivo verificar se os três grupos (controle, perdas leves e perdas moderadas) eram estatisticamente diferentes sob o ponto de vista das amplitudes das EOAPD.

A análise foi realizada pela Análise de Variância de Kruskal-Wallis. Os grupos que diferem entre si, identificados pelo teste de comparações múltiplas, foram destacados na coluna de "diferenças significantes", ao nível de $5 \%$.

Foi observada pela Análise de Variância de Kruskal-Wallis, a existência de diferenças significantes (demonstrado por asterisco) nas amplitudes 
Tabela 1 - Relação entre as frequências na audiometria tonal por via aérea e das EOAPD

\begin{tabular}{cc}
\hline Via Aérea & EOAPD \\
\hline $2.000 \mathrm{~Hz}$ & $1797 \mathrm{~Hz}$ \\
$3.000 \mathrm{~Hz}$ & $2.566 \mathrm{~Hz}$ \\
$4.000 \mathrm{~Hz}$ & $3.640 \mathrm{~Hz}$ \\
$6.000 \mathrm{~Hz}$ & $5.133 \mathrm{~Hz}$ \\
$8.000 \mathrm{~Hz}$ & $7.280 \mathrm{~Hz}$ \\
\hline
\end{tabular}

das EOAPD do protocolo T1 e T2 para todas as cinco frequências.

Com relação aos dados de $\mathrm{T} 1$, observou-se que o $\mathrm{G} 1$ apresentou amplitudes significantemente maiores que os G2 e G3. O grupo com perdas auditivas neurossensoriais de graus leve (G2) apresentou amplitudes nas frequências $1.797(2.000 \mathrm{~Hz})$, $3.640(4.000 \mathrm{~Hz})$ e $5.133 \mathrm{~Hz}(6.000 \mathrm{~Hz})$, significantemente maiores que o grupo com perdas neurossensoriais de grau moderado (G3).

$\mathrm{Na}$ análise do protocolo T2, observou-se a existência de diferenças significantes (demonstrados por asterisco) nas amplitudes das EOAPD para as frequências de $1.797(2.000 \mathrm{~Hz}), 2.566$ $(3.000 \mathrm{~Hz}), 3.649(4.000 \mathrm{~Hz})$ e $5.133 \mathrm{~Hz}(6.000 \mathrm{~Hz})$ entre os grupos. O G1 (controle) apresentou amplitudes significantemente maiores que os grupos $\mathrm{G} 2$ e G3. O grupo com perdas neurossensoriais de grau leve (G2) apresentou amplitudes nas frequências $1.797(2.000 \mathrm{~Hz}), 3.640(4.000 \mathrm{~Hz})$ e $5.133 \mathrm{~Hz}$ $(6.000 \mathrm{~Hz})$ significantemente maiores que os grupos com perdas auditivas neurossensoriais de grau moderado.

Foi avaliada a existência de variação significante das amplitudes das EOAPD entre os protocolos T1 e T2. Esta análise teve como objetivo verificar se existe redução significante nas amplitudes das EOAPD nos protocolos T1 de forma comparativa a T2, para cada grupo estudado (G1, G2 e G3).

Para realizar essa avaliação foi analisada a variação absoluta do T1 para T2 obtido pela seguinte fórmula:

$$
\begin{gathered}
\text { Var abs no } 1797 \mathrm{~Hz}=1797 \mathrm{~Hz} \\
\text { no T2 }-1797 \mathrm{~Hz} \text { no T1 }
\end{gathered}
$$

A Tabela 3 fornece as médias, erros padrões (EP) e o correspondente nível descritivo ( $p$ valor) do teste estatístico para o grupo controle (G1), grupos com perdas auditivas neurossensoriais de grau leve (G2) e perdas auditivas neurossensoriais de grau moderado (G3), respectivamente. A análise estatística foi realizada pelo teste não paramétrico de Wilcoxon.
De acordo com a Tabela 3, observa-se para o grupo controle G1 a existência de um aumento significante (conforme marcado com asterisco) do T1 para T2 (variação positiva) nas amplitudes das frequências $2.566 \mathrm{~Hz}(p=0,0003)$ e $3.640 \mathrm{~Hz}$ $(p=0,0003)$. Em média, observou-se um aumento entre 4 a $5 \mathrm{~dB}$ do T1 para T2. Para o grupo G2, houve um aumento significante do T1 para T2 nas amplitudes de todas as frequências no grupo com perda auditiva neurossensorial de grau leve. Em média, observou-se um aumento entre 3 a $5 \mathrm{~dB}$ do T1 para T2. Finalmente, para o grupo G3 a existência de um aumento significante de T1 para T2 nas amplitudes das frequências de $1.797 \mathrm{~Hz}(p=0,009)$, $2.566 \mathrm{~Hz}(p=0,0005)$ e $7.280 \mathrm{~Hz}(p=0,0001)$ no grupo com perdas auditivas neurossensoriais de grau moderado. Em média, observou-se um aumento entre 5 a 8 dB do T1 para T2.

\section{DISCUSSÃO}

\section{Análise das amplitudes das EOAPD nos grupos G1, G2 e G3}

Analisando a Tabela 2, encontrou-se que no protocolo $\mathrm{T} 1$ houve diferenças significantes nas amplitudes das EOAPD para todas as cinco frequências (1.797 Hz; $2.560 \mathrm{~Hz} ; 3.640 \mathrm{~Hz} ; 5.133 \mathrm{~Hz}$ e 7.282 $\mathrm{Hz})$ e, com relação ao protocolo $\mathrm{T} 2(\mathrm{~L} 1=\mathrm{L} 2=70$ dB NPS), observou-se a existência de diferenças significantes somente para as frequências: 1.797 $\mathrm{Hz}, 2.566 \mathrm{~Hz}, 3.649 \mathrm{~Hz}$ e $5.133 \mathrm{~Hz}$. Quanto maior o grau da perda auditiva, menor a amplitude, constatada na coluna de "diferenças significantes" para a maioria das frequências, em ambos os protocolos.

Estudando a diferença das amplitudes em indivíduos com audição dentro da normalidade e indivíduos com perdas auditivas neurossensoriais, encontrou-se uma diminuição significante em relação ao grupo de indivíduos com audição dentro da normalidade (grupo controle) e indivíduos com perdas auditivas neurossensoriais ${ }^{6,14}$ corroborando esta pesquisa. Provavelmente deve haver maior quantidade de células ciliadas preservadas nos indivíduos com audição dentro da normalidade, em relação aos indivíduos com perdas auditivas neurossensoriais. Outros referiram, ainda, que essa diferença era verificada principalmente nas frequências altas, de 2.000 a $6.000 \mathrm{~Hz}$, como visto nas frequências mais altas, acima de $8.000 \mathrm{~Hz}{ }^{6,15}$.

Isso ocorre porque nas frequências baixas, há interferência tanto de ruídos externos como os ruídos ambientais, ruídos internos como o da respiração bem como a não necessidade de um alto falante com maior voltagem para que produza a distorção. 
Tabela 2 - Análise estatística das amplitudes das EOAPD do protocolo T1 e T2 segundo o grupo estudado (G1, G2 e G3)

\begin{tabular}{|c|c|c|c|c|c|c|}
\hline \multirow{16}{*}{$\mathrm{T} 1$} & Amplitude & Grupo & Média & D.P. & $p$ valor & \# significante \\
\hline & $1797 \mathrm{~Hz}$ & G1 & 16,02 & 6,34 & $0,0001^{*}$ & normal leve \\
\hline & $\sim 2000 \mathrm{~Hz}$ & G2 & 7,44 & 6,06 & & normal mod \\
\hline & & G3 & 2,61 & 6,88 & & leve mod \\
\hline & $2566 \mathrm{~Hz}$ & G1 & 11,84 & 6,01 & $0,0001^{*}$ & normal leve \\
\hline & $\sim 3000 \mathrm{~Hz}$ & G2 & 4,78 & 5,19 & & normal mod \\
\hline & & G3 & 2,15 & 3,72 & & \\
\hline & $3640 \mathrm{~Hz}$ & G1 & 14,63 & 4,68 & $0,0001^{*}$ & normal leve \\
\hline & $\sim 4000 \mathrm{~Hz}$ & G2 & 5,23 & 6,01 & & normal mod \\
\hline & & G3 & 0,97 & 3,79 & & leve mod \\
\hline & $5133 \mathrm{~Hz}$ & G1 & 17,89 & 8,61 & $0,0001^{*}$ & normal leve \\
\hline & $\sim 6000 \mathrm{~Hz}$ & G2 & 5,54 & 7,22 & & normal mod \\
\hline & & G3 & 0,26 & 3,35 & & leve mod \\
\hline & $7280 \mathrm{~Hz}$ & G1 & 16,03 & 8,96 & $0,0001^{*}$ & normal leve \\
\hline & $\sim 8000 \mathrm{~Hz}$ & G2 & 8,47 & 7,08 & & normal mod \\
\hline & & G3 & 4,88 & 4,84 & & \\
\hline \multirow{16}{*}{$\mathrm{T} 2$} & Amplitude & Grupo & Média & D.P. & $p$ valor & \# significante \\
\hline & $1797 \mathrm{~Hz}$ & G1 & 16,7 & 5,24 & $0,0001^{*}$ & normal leve \\
\hline & $\sim 2000 \mathrm{~Hz}$ & G2 & 13,11 & 4,86 & & normal mod \\
\hline & & G3 & 8,29 & 5,18 & & leve mod \\
\hline & $2566 \mathrm{~Hz}$ & G1 & 16,3 & 7,34 & $0,0001^{*}$ & normal leve \\
\hline & $\sim 3000 \mathrm{~Hz}$ & G2 & 9,61 & 5,58 & & normal mod \\
\hline & & G3 & 6,72 & 4,46 & & \\
\hline & $3640 \mathrm{~Hz}$ & G1 & 19,56 & 7,59 & $0,0001^{*}$ & normal leve \\
\hline & $\sim 4000 \mathrm{~Hz}$ & G2 & 9,45 & 7,33 & & normal mod \\
\hline & & G3 & 2,76 & 5,01 & & leve mod \\
\hline & $5133 \mathrm{~Hz}$ & G1 & 18,89 & 8,12 & $0,0001^{*}$ & normal leve \\
\hline & $\sim 6000 \mathrm{~Hz}$ & G2 & 9,54 & 9,99 & & normal mod \\
\hline & & G3 & 2,3 & 3,79 & & leve mod \\
\hline & $7280 \mathrm{~Hz}$ & G1 & 16,17 & 7,51 & 0,18 & \\
\hline & $\sim 8000 \mathrm{~Hz}$ & G2 & 12,2 & 6,14 & & \\
\hline & & G3 & 12,79 & 4,04 & & \\
\hline
\end{tabular}

D.P.: Desvio Padrão

Utilizando EOAPD, a fim de comparar as respostas encontradas em pacientes com perdas auditivas neurossensoriais e em indivíduos com audição dentro dos limites da normalidade, com o protocolo $\mathrm{L} 1=65 \mathrm{~dB}$ NPS e L2 $=50 \mathrm{~dB}$ NPS, os autores puderam observar que as amplitudes das EOAPD foram capazes de distinguir as orelhas normais daquelas com deficiências auditivas, nas frequências de 4.000 a $8.000 \mathrm{~Hz}$ e, de forma mais discreta, em $2.000 \mathrm{~Hz}^{6}$. Já outros autores analisaram indivíduos com perdas auditivas e indivíduos com audição dentro da normalidade, relataram não ser possível correlacionar as amplitudes das respostas para pre- dizer a perda auditiva, mas as EOAPD apresentaram uma correlação significante com o limiar auditivo, principalmente na frequência de $1.000 \mathrm{~Hz}{ }^{14}$. Com relação a esta pesquisa, houve diferenças significantes nas frequências de 2.000, 3.000, $4.000 \mathrm{e}$ $6.000 \mathrm{~Hz}$, quando comparados os grupos controle, o com perdas auditivas neurossensoriais de grau leve e o com perdas auditivas neurossensoriais de grau moderado, mas foram encontrados valores mínimos e máximos com grande variação nos três grupos, corroborando os achados do estudo ${ }^{14}$, não se pode predizer o grau das perdas auditivas a partir das amplitudes encontradas nas EOAPD. 
Tabela 3 - Análise estatística das amplitudes do T1 e T2 nos grupos G1,G2 e G3

\begin{tabular}{|c|c|c|c|c|}
\hline Grupos & $\begin{array}{c}\text { Variação Absoluta } \\
\text { (T2 - T1) }\end{array}$ & Média & E.P. & $p$ valor \\
\hline \multirow{5}{*}{ G1 } & $1797 \mathrm{~Hz}$ & 0,67 & 1,3 & 0,98 \\
\hline & $2566 \mathrm{~Hz}$ & 4,47 & 1,07 & $0,0003^{*}$ \\
\hline & $3640 \mathrm{~Hz}$ & 4,93 & 1,16 & $0,0003^{*}$ \\
\hline & $5133 \mathrm{~Hz}$ & 1 & 0,91 & 0,32 \\
\hline & $7280 \mathrm{~Hz}$ & 0,13 & 1,11 & 0,82 \\
\hline \multirow{5}{*}{ G2 } & $1797 \mathrm{~Hz}$ & 5,67 & 1,39 & $0,0001^{*}$ \\
\hline & $2566 \mathrm{~Hz}$ & 4,83 & 1,14 & $0,0003^{*}$ \\
\hline & $3640 \mathrm{~Hz}$ & 4,22 & 0,97 & $0,0003^{*}$ \\
\hline & $5133 \mathrm{~Hz}$ & 4 & 1,28 & $0,001^{*}$ \\
\hline & $7280 \mathrm{~Hz}$ & 3,73 & 0,95 & $0,0007^{*}$ \\
\hline \multirow{5}{*}{ G3 } & $1797 \mathrm{~Hz}$ & 5,68 & 1,9 & $0,009^{*}$ \\
\hline & $2566 \mathrm{~Hz}$ & 4,57 & 1,07 & $0,0005^{*}$ \\
\hline & $3640 \mathrm{~Hz}$ & 1,8 & 1,1 & 0,17 \\
\hline & $5133 \mathrm{~Hz}$ & 2,05 & 0,92 & 0,063 \\
\hline & $7280 \mathrm{~Hz}$ & 7,91 & 0,84 & $0,0001^{*}$ \\
\hline
\end{tabular}

E.P.: Erro Padrão

\section{Análise das amplitudes das EOAPD dos T1 e T2 nos grupos G1, G2 e G3}

Pesquisando a literatura, em estudo similar, os autores utilizaram dois protocolos diferentes, o primeiro com intensidades de $\mathrm{L} 1=50 \mathrm{~dB}$ NPS e de $\mathrm{L} 2$ $=65 \mathrm{~dB}$ NPS e o segundo com intensidades iguais para L1 e L2, isto é $65 \mathrm{~dB}$ NPS. Encontraram para os dois protocolos uma correlação significante entre os limiares tonais e os resultados das EOAPD, mas para o segundo protocolo houve um melhor desempenho, apresentando maiores amplitudes ${ }^{16}$. Neste trabalho, também foram observadas maiores amplitudes no T2 ( $\mathrm{L} 1=\mathrm{L} 2=70 \mathrm{~dB}$ NPS) em relação ao T1 $(\mathrm{L} 1=65 \mathrm{~dB}$ NPS e L2 $=55 \mathrm{~dB}$ NPS) em praticamente todas as frequências testadas. Avaliando 80 trabalhadores de indústria têxtil, com resultados da audiometria tonal normais, a autora também encontrou menor ocorrência de amplitude das EOA em trabalhadores do que no grupo controle, principalmente nas frequências altas, não observou diferenças significantes nas EOAPD em relação ao nível de pressão sonora dos tons puros, utilizando os protocolos $\mathrm{L} 1=\mathrm{L} 2=70 \mathrm{dBNPS}$ e $\mathrm{L} 1>\mathrm{L} 2$ (65 / 55 dBNPS) ${ }^{17}$, provavelmente pela alteração que o ruído pode provocar nas células ciliadas externas, mesmo sem apresentar alteração auditiva.
Portanto, observa-se a existência de um aumento significante do T1 para T2 (variação positiva) nas amplitudes das EOAPD:

- das frequências $2.566 \mathrm{~Hz}(p=0,0003)$ e $3.640 \mathrm{~Hz}$ $(p=0,0003)$ para o grupo controle (G1).

- de todas as frequências nos indivíduos com perda auditiva neurossensorial de grau leve (G2).

- das frequências $1.797 \mathrm{~Hz}(p=0,009), 2.566 \mathrm{~Hz}$ $(p=0,0005)$ e $7.280 \mathrm{~Hz}(p=0,0001)$ nos indivíduos com perda auditiva neurossensorial de grau moderado (G3).

\section{CONCLUSÃO}

Com relação ao grau da perda auditiva, pode-se concluir que, quanto maior a perda auditiva, menor o aumento da amplitude das EOAPD.

Com relação aos protocolos T1 e T2, foi possível observar um melhor desempenho do protocolo L1 = $\mathrm{L} 2=70 \mathrm{~dB}$ NPS em relação ao protocolo $\mathrm{L} 1=65$ $\mathrm{dB}$ NPS e L2 = $55 \mathrm{~dB}$ NPS, apresentando amplitudes maiores na maioria das frequências nos três grupos. 


\section{ABSTRACT}

Purpose: to comparatively study the amplitudes of otoacoustic emission distortion product in normal hearing with mild and moderate sensorineural hearing loss. Methods: forty-one adults, 25 female and 16 male ranging from 18 to 25 years old took part in the study. Pure tone and speech audiometry were performed in order to separate the groups, according to their audiometric profile, impedance audiometries were performed in order to evaluate the middle ear conditions and distortion product otoacoustic emission were using T1 $(\mathrm{L} 1=65 \mathrm{dBSPL}$ and $\mathrm{L} 2=55 \mathrm{dBNSPL})$ and T2 (L1=L2=7-dBSPL) protocols. Sixty-three ears were examined (32 right ear and 31 left ear). Selected subjects were distributed into three groups: G1 including 21 subjects with normal pure tone and speech audiometry (control group), G2 including 21 subjects with mild sensorineural hearing losses and G3 including 21 subjects with moderate sensorineural hearing impaired. The results of pure tone and speech audiometry were compared with the results of DPOAE. Results: there is evidence of reduced amplitude or absence of response in the group with mild hearing loss (G2) and absence of response in the group with moderate hearing loss (G3). Conclusion: these findings suggest that the amplitudes of DPOAE decreased in agreement with the increase hearing loss level.

KEYWORDS: Hearing Loss; Amplitude; Otoacoustic Emissions, Spontaneous

\section{REFERÊNCIAS}

1. Gravel JS, Hood LJ. Avaliação audiológica infantil. In: Musiek FE, Rintelmann WF. Perspectivas atuais em avaliação auditiva. São Paulo: Manole; 2001. p. 301-32.

2. Kemp DT, Ryan S, Bray P. A guide to the effective use of otoacoustic emissions. Ear Hear. 1990; 11(2):93-105.

3. Pinto VS, Lewis DR. Emissões otoacústicas: produto de distorção em lactentes até dois meses de idade. Pró-Fono. 2007; 19(2):195-204.

4. Fiorini AC, Parrado-Moran MES. Emissões otoacústicas - produto de distorção: estudo de diferentes relações de níveis sonoros no teste em indivíduos com e sem perdas auditivas. Dist Comun. 2005; 17(3):385-96.

5. Bento RF, Miniti A, Marone SAM. Tratado de Otologia. São Paulo: Edusp; 1998; p. 112-6.

6. Gorga MP, Neely ST, Bergman B, Beauchaine KL, Kaminski JR, Peters J, et al. Otoacoustic emissions from normal-hearing and hearing-impaired subjects: distortion product responses. J Acoustic Soc Am. 1993; 93(4Pt1):2050-60.

7. Basseto MCA, Chiari BM, Azevedo MF. Emissöes otoacústicas evocadas transientes (EOAET): amplitude da resposta em recém-nascidos a termo e pré-termo. Rev Bras Otorrinolaringol. 2003; 69(1):84-92.

8. Gkoritsa E, Korres S, Psarommatis I, Tsakanikos M, Apostolopoulos N, Ferekidis E. Maturation of the auditory system: 1 . Transient otoacoustic emissions as an index of inner ear maturation. Int $\mathrm{J}$ Audiol. 2007; 46(6):271-6.

9. Marone MR. Emissões otoacústicas produto de distorção em recém-nascidos medicados com ototóxicos São Paulo. [tese]. São Paulo (SP): Universidade de São Paulo; 2006.

10. Ferreira LLA. Amplitude das emissões otoacústicas por produto de distorção em motociclistas normo-ouvintes. [tese] São Paulo (SP): Universidade de São Paulo; 2005.

11. Lapsley Miller JA, Marshall L, Heller LM. A longitudinal study of changes in evoked otoacoustic emissions and pure-tone thresholds as measured in a hearing conservation program. Int J Audiol. 2004; 43(6):307-22.

12. Biro K, Nnoszek L, Prekopp P, Nagyiványi K, Géczi L, Gaudi, I et al. Characteristics and risk factors of cisplatin-induced ototoxicity in testicular cancer patients detected by distorsion product otoacoustic emission. Oncol. 2006; 70(3):177-84.

13. Frota S. Fundamentos em fonoaudiologia: audiologia. Rio de Janeiro: Guanabara Koogan; 2003.

14. Moulin A, Bera JC, Collet L. Distortion product otoacoustic emissions and sensorineural hearing loss. Audiology. 1994; 33(6):305-26.

15. Norton SJ, Gorga MP, Widen JE, Folsom RC, Sininger $\mathrm{Y}$, Cone-Wesson $\mathrm{B}$, et al. Identification of neonatal hearing impairment: evaluation of transient evoked otoacoustic emission, distortion product otoacoustic emission, and auditory brain stem response test performance. Ear Hear. 2000; 21(5):508-28. 
16. Sun X-M, Kim DO, Jung MD, Randolph KJ. Distortion product otoacoustic emission test of sensorineural hearing loss in humans: comparison of unequal- and equal-level stimuli. Ann Otol Rhinol Laryngol. 1996; 105(12):982-90.
17. Fiorini $A C$. $O$ uso de registros de emissões otoacústicas como instrumento de vigilância epidemiológica de alterações auditivas em trabalhadores expostos a ruído. [tese] São Paulo (SP): Universidade de São Paulo; 2000.

RECEBIDO EM: 12/09/2008

ACEITO EM: 23/04/2009

Endereço para correspondência:

Maria Isabel Kós

Rua Timóteo da Costa, 1033 bl3 ap. 903

Rio de Janeiro - RJ

CEP: 22450-130

E-mail: bila@kos.med.br 